\title{
A Hybrid Approach for Fault Tree Analysis Combining Probabilistic Method with Fuzzy Numbers
}

\author{
Julwan H. Purba ${ }^{1}$, Jie Lu ${ }^{1}$, Da Ruan ${ }^{2}$, and Guangquan Zhang ${ }^{1}$ \\ 1 University of Technology, Sydney (UTS) \\ P.O. Box 123, Broadway, NSW 2007, Australia \\ \{julwan, jielu, zhangg\}@it.uts.edu.au \\ 2 Belgian Nuclear Research Centre (SCK•CEN) \\ Boeretang 200, 2400 Mol, Belgium \\ druan@sckcen.be
}

\begin{abstract}
Conventional fault tree analysis in safety analysis of complex engineering systems calculates the occurrence probability of the top undesired event using probabilistic failure rates. However, it is often very difficult to obtain those failure rates well in advance due to insufficient data, environment changing or new components. Fuzzy numbers can be applied to estimate failure rates by handling linguistic terms. This study proposes a hybrid approach of Fuzzy Numbers and Fault Tree Analysis to solve the conventional problem and describes its procedures using a case study of emergency core cooling system of a typical nuclear power plant.

Key words:Fault tree analysis, probabilistic failure rate, fuzzy failure rate, safety analysis
\end{abstract}

\section{Introduction}

Fault Tree Analysis (FTA) is widely used for safety analysis of complex engineering systems such as nuclear power plants (NPPs). Conventional FTA utilizes failure rates of every individual basic event constructing the tree to approximately calculate the occurrence probability of the top undesired event. However, it is often very difficult to exactly estimate the basic event failure rates due to insufficient data, environment changing or new components.

Fuzzy probabilities have been introduced to calculate the failure probability of a typical emergency cooling system using FTA [1]. The occurrence probability of the top undesired event is calculated using fuzzy multiplication rules for the Boolean AND gate and fuzzy complementation rules for the Boolean OR gate. However, this approach is limited only for trapezoidal fuzzy numbers. It also does not consider qualitative analysis and criticality analysis. The $\alpha-$ cut method has been introduced to calculate the failure probability of the reactor protective system (WASH-1400) [2]. All basic events are assumed to have probability distributions prior to designing triangular fuzzy numbers. The point 
median value and the error factor are used to represent the lower bound and the upper bound of the triangular fuzzy numbers. The middle value of the triangular fuzzy numbers is represented by the point median value. However, this approach does not consider qualitative analysis prior to estimating the occurence probability of the top event. A computational system analysis for FTA called FuzzyFTA has been developed and implemented to calculate the failure probability of Auxiliary Feedwater System (AFWS) of Angra-I Westinghouse NPP [3] and containment cooling system (CCS) of a typical four-loops pressurized water reactor [4]. However, this methodology is applicable only for triangular fuzzy numbers.

This paper proposes a Fuzzy Numbers based Fault Tree Analysis (FNFTA) approach. The approach combines probabilistic method with fuzzy numbers to estimate the failure probability of the top event. Fuzzy numbers can be in triangular and/or in trapezoidal form. The paper is organized as follows. The fuzzy numbers, fuzzy possibility scores and failure rates are explained in Section 2. Section 3 discusses the structure of the FNFTA approach and its procedures. In Section 4, a case study for NPPs shows the applicability of the approach. Finally, Section 5 summarizes future research tasks.

\section{Fuzzy Numbers, Fuzzy Possibility Scores and Failure Rates}

\subsection{Fuzzy Numbers}

A fuzzy number $A$ is a subset of real line $R$ whose membership function $f_{A}(x)$ can be continuously mapping from $R$ into a closed interval $[0, w]$ where $0 \leq w \leq 1$ [5]. A normal fuzzy number $A$ is expressed as follows.

$$
f_{A}(x)= \begin{cases}f_{A}^{L}(x), & a \leq x \leq b \\ 1, & b \leq x \leq c \\ f_{A}^{R}(x), & c \leq x \leq d \\ 0, & \text { otherwise }\end{cases}
$$

where $f_{A}^{L}(x):[a, b] \rightarrow[0,1]$ and $f_{A}^{R}(x):[c, d] \rightarrow[0,1]$. If both $f_{A}^{L}(x)$ and $f_{A}^{R}(x)$ are linear, then the fuzzy number $A$ is a trapezoidal fuzzy number and usually denoted by $A=(a, b, c, d)$. When $b=c$, the trapezoidal fuzzy number becomes the triangular fuzzy number.

\subsection{Fuzzy Possibility Scores}

Fuzzy possibility score (FPS) is a crisp score that represents experts belief of the most likely score that an event may occur [6][7]. Centroid-based distance method is to convert fuzzy numbers into fuzzy possibility scores [8]. The centroid $\left(x_{0}, y_{0}\right)$ of the fuzzy number $A$ is calculated as follows.

$$
x_{0}(A)=\frac{\int_{a}^{b}\left(x \cdot f_{A}^{L}(x)\right) d x+\int_{b}^{c} x \cdot d x+\int_{c}^{d}\left(x \cdot f_{A}^{R}(x)\right) d x}{\int_{a}^{b} f_{A}^{L}(x) d x+\int_{b}^{c} d x+\int_{c}^{d} f_{A}^{R}(x) d x}
$$




$$
y_{0}(A)=\frac{\int_{0}^{1}\left(y \cdot g_{A}^{R}(y)\right) d y-\int_{0}^{1}\left(y \cdot g_{A}^{L}(y)\right) d y}{\int_{0}^{1} g_{A}^{R}(y) d y-\int_{0}^{1} g_{A}^{L}(y) d y}
$$

where $g_{A}^{R}(y)$ and $g_{A}^{L}(y)$ are the inverse function of $f_{A}^{R}(x)$ and $f_{A}^{L}(x)$, respectively.

The FPS of fuzzy number $A$ is the Euclidean distance of fuzzy number $A$, which is calculated from the origin to the centroid of the fuzzy numbers as follows.

$$
F P S(A)=\sqrt{\left(x_{0}\right)^{2}+\left(y_{0}\right)^{2}}
$$

\subsection{Fuzzy Failure Rates}

Fuzzy failure rate (FFR) is an error rate which is obtained by dividing the frequency of an error with the total chance that an event may have error [7]. Onisawa proposed a logarithmic function to estimate the nature of human justification in [9]:

$$
e=\frac{1}{1+\left(K \times \log \left(1 / E_{m}\right)^{3}\right)}
$$

where $e$ is analogous to FPS and $E_{m}$ is the most likely fault rate. $K$ is a constant, which represents the safety criterion based on the lowest lower bound of the error rate and error rates of a routine. Onisawa defined that $K=2.301$. $E_{m}$ is represented by FFR, which is estimated in [10]:

$$
F F R= \begin{cases}\frac{1}{10^{m}}, & F P S \neq 0 \\ 0, & F P S=0\end{cases}
$$

where

$$
m=\left[\frac{1-F P S}{F P S}\right]^{\frac{1}{3}} \times 2.301
$$

\section{The Structure of the FNFTA Approach}

The FNFTA approach consists of four analysis phases, which are system analysis phase, qualitative analysis phase, quantitative analysis phase, and criticality analysis phase (Fig. 1).

Phase 1: System Analysis Phase. System performance is analysed to build a fault tree describing failure modes that may occur to the system during its life time. This output becomes input to the second phase.

Phase 2: Qualitative Analysis Phase. Repeating events are removed from the fault tree by identifying cut sets and minimal cut sets. The output of this phase is a simplified fault tree which is equivalent to the fault tree obtained in the first phase but it is free from repeating events. This output becomes input to the third phase. Figure 2 shows the flowchart of this phase. 


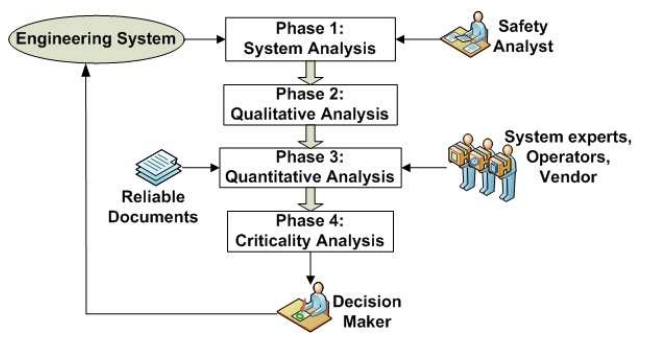

Fig. 1. The structure of the FNFTA approach

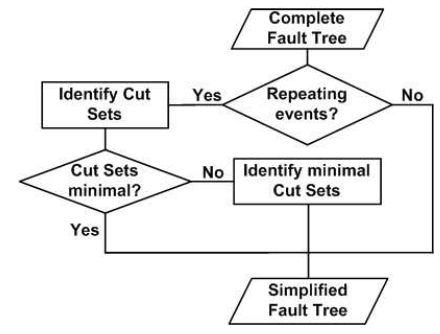

Fig. 2. The flowchart for qualitative analysis phase

Phase 3: Quantitative Analysis Phase. All basic events constructing the simplified fault tree are divided into two different evaluation techniques; probabilistic method and fuzzy numbers. The failure rates of basic events, which are evaluated using probabilistic method, can be obtained from reliable documents. The failure rates of other basic events are estimated using fuzzy numbers, which are designed based on expert justifications. These fuzzy numbers then are converted into FPS and finally into FFR. After all basic events have failure rates, Boolean algebra is used to estimate the occurrence probability of the top undesired event. The occurrence probability of the top event for two independent events for OR gate and AND gate are given in [11].

$$
\begin{gathered}
P_{T}=P_{A}+P_{B}-P_{A} \times P_{B} \\
P_{T}=P_{A} \times P_{B}
\end{gathered}
$$

where $\mathrm{P}_{A}$ and $\mathrm{P}_{B}$ are the failure rates of basic event $\mathrm{A}$ and basic event $\mathrm{B}$, respectively. The output of this phase is the occurrence probability of the top undesired event and is an input to the fourth phase. Figure 3 shows the flowchart of this phase.

Phase 4: Criticality Analysis Phase. This phase evaluates how far a basic event contributes to the occurrence of the top undesired event by calculating the criticality index of every basic event. Based on the calculated criticality index, the order of critical components can be justified. Fussell-Vesely (FV) importance 


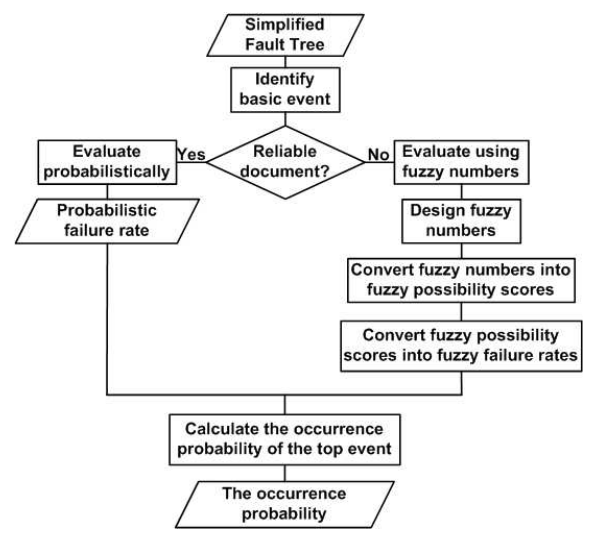

Fig. 3. The flowchart for quantitative analysis phase

algorithm as shown in (8) can be used to calculate the criticality index of a component [12].

$$
F V_{i}=\frac{R_{0}-R_{i}^{-}}{R_{0}}
$$

where $R_{0}$ is the probability of the top event for overall basic events and $R_{i}^{-}$is the probability of the top event by setting the probability of basic event $i$ to 0 . Decision makers use this criticality index to improve the safety features of the analyzed NPP. Figure 4 shows the flowchart of this phase.

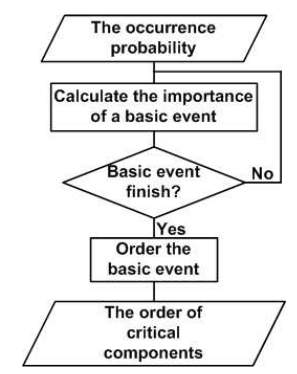

Fig. 4. The flowchart for criticality analysis phase

\section{A Case Study for Nuclear Power Plants}

The failure of a typical emergency core cooling system (ECCS) which is adopted from [1] is used to demonstrate the applicability of this approach. ECCS works to mitigate the consequences of a loss of coolant accident (LOCA). There are two 
operation modes of this ECCS, automatic operation and manual operation. The manual operation will only work when the automatic operation fails to mitigate the accident. Further details on this ECCS operation can be found in [1].

Phase 1: System Analysis Phase. In this case study, only the failure scenario of the automatic operation is analyzed. The fault tree describing the failure scenario of this operation mode is shown in Fig. 5. The top undesired event is the failure of automatic ECCS (FAECCS).

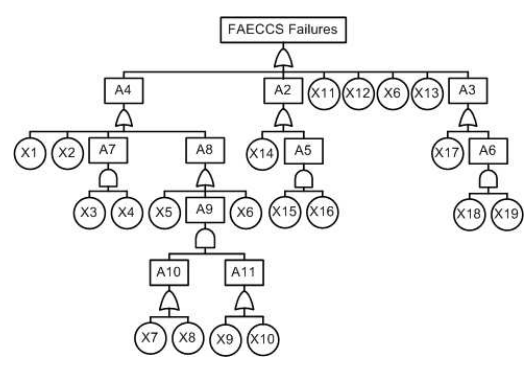

Fig. 5. The fault tree for FAECCS

Phase 2: Qualitative Analysis Phase. FAECCS in Fig. 5 still has one repeating event, which is a basic event X6. This repeating event can be removed using the combination of MOCUS algorithm and the rules of Boolean algebra to obtain cut sets and minimal cut sets [13].

The MOCUS algorithm evaluates events starting from the top events down to the bottom events. Every intermediate events found in the evaluation process will be substituted by the lower events. In Fig. 5, FAECCS can be represented by $(\mathrm{A} 4+\mathrm{A} 2+\mathrm{X} 11+\mathrm{X} 12+\mathrm{X} 6+\mathrm{X} 13+\mathrm{A} 3)$ where $\mathrm{A} 4, \mathrm{~A} 2$ and $\mathrm{A} 3$ are intermediate events. A4 then can be represented by $(\mathrm{X} 1+\mathrm{X} 2+\mathrm{A} 7+\mathrm{A} 8)$, $\mathrm{A} 2$ by $(\mathrm{X} 14+\mathrm{A} 5)$ and A3 by $(\mathrm{X} 17+\mathrm{A} 6)$. Therefore the FAECCS can be represented by $(\mathrm{X} 1+\mathrm{X} 2+\mathrm{A} 7+\mathrm{A} 8+\mathrm{X} 14+\mathrm{A} 5+\mathrm{X} 11+\mathrm{X} 12+\mathrm{X} 6+$ $\mathrm{X} 13+\mathrm{X} 17+\mathrm{A} 6)$. Using the same procedures for the next intermediate events found and by implementing the law of Boolean algebra absorption, the final representation of FAECCS are $(\mathrm{X} 1+\mathrm{X} 2+(\mathrm{X} 3 . \mathrm{X} 4)+\mathrm{X} 5+((\mathrm{X} 7+\mathrm{X} 8)$. (X9 $+\mathrm{X} 10))+\mathrm{X} 6+\mathrm{X} 14+(\mathrm{X} 15 . \mathrm{X} 16)+\mathrm{X} 11+\mathrm{X} 12+\mathrm{X} 13+\mathrm{X} 17+(\mathrm{X} 18 . \mathrm{X} 19))$ where symbol dot (.) represents AND gate and symbol plus $(+)$ represents OR gate in the tree. This final representation as depicted in Fig. 6 is a simplified fault tree of the fault tree in Fig. 5.

Phase 3: Quantitative Analysis Phase. In this phase, basic events are classified into two evaluation groups, which are probabilistic method and fuzzy numbers. For illustrative purposes only, we just simply evaluate basic events X1, X2, X3, $\mathrm{X} 4, \mathrm{X} 5, \mathrm{X} 6, \mathrm{X} 7, \mathrm{X} 8, \mathrm{X} 11$, and X12 using probabilistic method and all these 


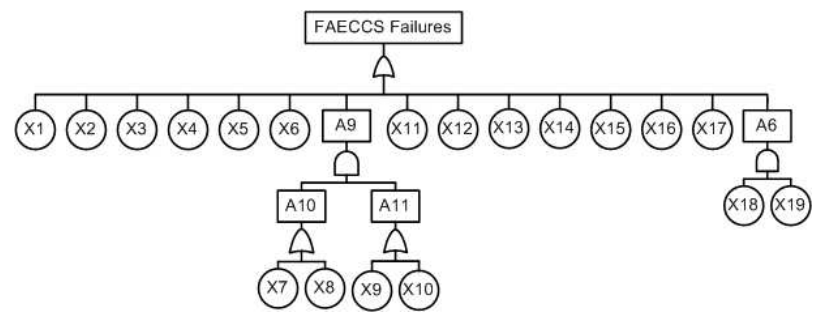

Fig. 6. The simplified fault tree

events are assumed to have probabilistic failure rates of $1.5 \times 10^{-4}$. Other basic events are evaluated using fuzzy numbers. Basic events X9, X10, X12, X13, and $\mathrm{X} 14$ are evaluated using triangular fuzzy numbers of $(0.1,0.15,0.25,0.3)$; meanwhile basic events X15, X16, X17, X18, and X19 are evaluated using trapezoidal fuzzy numbers of $(0.1,0.25,0.3)$.

By solving (1) and (2) and substitute $\mathrm{a}=0.1, \mathrm{~b}=0.15, \mathrm{c}=0.25$ and $\mathrm{d}$ $=0.3$ for trapezoidal fuzzy numbers, we obtain $x_{0}=0.2$ and $y_{0}=0.444444$. FPS is then calculated using (3) and the result is 0.48737 . Finally, this FPS is substituted into (5) to calculate FFR and the result is $4.57 \times 10^{-3}$. With the same procedures, FFR for the triangular fuzzy numbers is $2.275 \times 10^{-3}$.

Using (6), the occurrence probability of intermediate event A10 and A11 are $2.0 \times 10^{-4}$ and $4.54 \times 10^{-3}$, respectively. Using (7), the occurrence probability of intermediate event A9 is $1.36 \times 10^{-6}$. With the same procedures, the occurrence probability of FAECCS is $2.1414 \times 10^{-2}$.

Phase 4: Criticality Analysis Phase. Using (8), the FV importance of basic event $\mathrm{X} 1$ is:

$$
F V_{X 1}=\frac{0.021413921-0.02126711}{0.021413921}=6.8558 \times 10^{-3}
$$

The FV importances of other basic events in Table 1 are calculated using the same procedures. Basic events X15, X16 and X17 are the most critical compo-

Table 1. The FV importance and the critical order of basic events

\begin{tabular}{ccc}
\hline Component & FV importance Critical order \\
\hline X1,X2,X3,X4,X5,X6,X11 & $6.8558 \mathrm{E}-3$ & 3 \\
X7,X8,X9,X10 & $3.1149 \mathrm{E}-5$ & 5 \\
X12,X13,X14 & $1.0420 \mathrm{E}-1$ & 2 \\
X15,X16,X17 & $2.0980 \mathrm{E}-1$ & 1 \\
X18,X19 & $9.5443 \mathrm{E}-4$ & 4 \\
\hline
\end{tabular}

nents followed by basic events X12, X13 and X14. These basic events need to be redesigned or changed in order to improve the reliability of FAECCS. 


\section{$5 \quad$ Summary and Future Works}

Three advantages are gained from this FNFTA approach. (1) Probabilistic failure rate can be combined with fuzzy numbers to solve the limitation of the conventional FTA. (2) The calculation of the failure probability of the top undesired event is more accurate than the previous fuzzy approach because cut sets and minimal cut sets are evaluated first in the qualitative analysis phase prior to the quantitative analysis phase. (3) The approach can be used to estimate the critical components and therefore decision makers can redesign or change critical components to improve the safety features of the system being analyzed. For future works, the conversion functions from fuzzy numbers into FFR need to be investigated in real-case studies.

\section{Acknowledgement}

The work presented in this paper was partially supported by the Australian Research Council (ARC) Discovery Grant PD0880739.

\section{References}

1. Misra, K.B., Weber, G.G.: Use of fuzzy set theory for level-I studies in probabilistic risk assessment. Fuzzy Sets and Systems 37(2), 139-160 (1990)

2. Suresh, P.V., Babar A.K., Venkat Raj, V.: Uncertainty in fault tree analysis: A fuzzy approach. Fuzzy Sets and Systems 83(2), 135-141 (1996)

3. Guimaraes, A.C.F., Ebecken, N.F.F.: FuzzyFTA: A fuzzy fault tree system for uncertainty analysis. Annals of Nuclear Energy 26(6), 523-532 (1999)

4. Guimaraes, A.C.F., Lapa, C.M.F.: Parametric fuzzy study for effects analysis of age on PWR containment cooling system. Applied Soft Computing 8(1), 1562-1571 (2008)

5. Dubois, D., Prade, H.: Operations on fuzzy numbers. International Journal of Systems Science 9, 613-626 (1978)

6. Yuhua, D., Datao, Y.: Estimation of failure probability of oil and gas transmission pipelines by fuzzy fault tree analysis. Journal of Loss Prevention in the Process Industries 18, 83-88 (2005)

7. Lin, C.T., Wang, M.J.J.: Hybrid fault tree analysis using fuzzy sets. Reliability Engineering \& System Safety 58(3), 205-213 (1997)

8. Wang, Y.M., Yang, J.B., Xu, D.L., Chin, K.S.: On the centroids of fuzzy numbers. Fuzzy Sets and Systems 157(7), 919-926 (2006)

9. Onisawa, T.: An approach to human reliability in man-machine systems using error possibility. Fuzzy Sets and Systems 27(2), 87-103 (1988)

10. Pan, N.F., Wang, H.: Assessing failure of bridge construction using fuzzy fault tree analysis. IEEE International Conference on Fuzzy Systems and Knowledge Discovery, Haikou, China (2007)

11. Haimes, Y.Y.: Fault trees. Risk modeling, assessment and management, 525-569, John Wiley \& Sons, Inc., New Jersey (2004)

12. Vinod, G., Kushwaha, H.S., Verma, A.K., Srividya, A.: Importance measures in ranking piping components for risk informed in-service inspection. Reliability Engineering \& System Safety 80(2), 107-113 (2003)

13. Ericson, C.A.: Fault tree analysis. Hazard analysis techniques for system safety, 183-221, John Wiley \& Sons, Inc., Virginia (2005) 